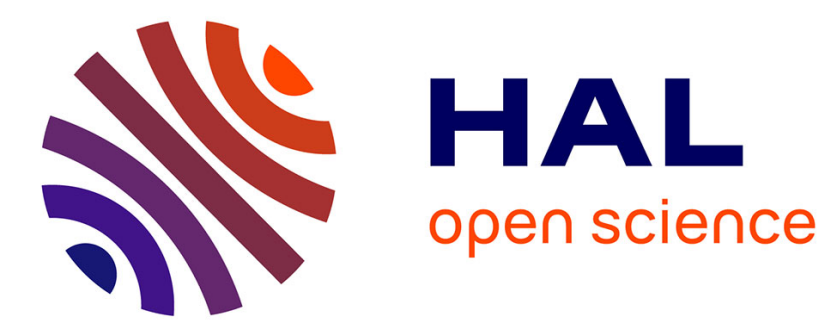

\title{
Non-Local Feedback Ramp Metering Controller Design
}

Denis Jacquet, Jonathan Jaglin, Damien Koenig, Carlos Canudas de Wit

\section{To cite this version:}

Denis Jacquet, Jonathan Jaglin, Damien Koenig, Carlos Canudas de Wit. Non-Local Feedback Ramp Metering Controller Design. Proceedings of the 11th IFAC Symposium on Control in Transportation Systems, CTS 2006, 2006, -, Netherlands. p. 6. hal-00098668

\section{HAL Id: hal-00098668 https://hal.science/hal-00098668}

Submitted on 25 Sep 2006

HAL is a multi-disciplinary open access archive for the deposit and dissemination of scientific research documents, whether they are published or not. The documents may come from teaching and research institutions in France or abroad, or from public or private research centers.
L'archive ouverte pluridisciplinaire HAL, est destinée au dépôt et à la diffusion de documents scientifiques de niveau recherche, publiés ou non, émanant des établissements d'enseignement et de recherche français ou étrangers, des laboratoires publics ou privés. 


\title{
NON-LOCAL FEEDBACK RAMP METERING CONTROLLER DESIGN
}

\author{
Denis Jacquet ${ }^{1}$, Jonathan Jaglin, Damien Koenig \\ and Carlos Canudas de Wit
}

\author{
Laboratoire d'Automatique de Grenoble \\ BP. 46, 38 402, St. Martin d'Hères, France
}

\begin{abstract}
This paper treats the problem of controlling large freeway systems through ramp metering to enhance their efficiency. Following a rigorous discretization methodology, an extension of the CTM model to inhomogeneous freeways with on and off ramps is proposed. Based on passivity theory and the piecewise affine formulation of this model, an LMI formulation is developed to design non-local feedback ramp metering controllers. Copyright 2006 IFAC.
\end{abstract}

Keywords: Freeway model, ramp metering, switched systems, LMI.

\section{INTRODUCTION}

Freeway traffic congestion has a high social and economical cost that tends to increase dramatically in large cities. Since the field studies on ramp metering efficiency reported in (Papageorgiou et al., 1997) and (Levinson and Zhang, 2005), traffic responsive metering strategies are considered to be appropriate to tackle the problem of excess demand on freeways. This article proposes a novel control approach based on a macroscopic traffic model to design dynamical and non-local metering controllers.

There is an abondant literature on equilibrium homogeneous macroscopic traffic models from the pioneering LWR model (Lighthill and Whitham, 1955) to the more recent Cell Transmission Model (CTM) proposed in (Daganzo, 1994). Extensions to inhomogeneous roads such as freeway sections with variable numbers of lanes were treated in (Lebacque, 1996). We propose in this paper an extension of the CTM model to include on/off ramps. The resulting model has a form similar to the CTM model, making it suitable for controller

\footnotetext{
1 Corresponding author: denis.jacquet@lag.ensieg.inpg.fr
}

design. This Inhomogeneous-CTM (I-CTM) has a complex solution structure involving hybrid dynamics due to the interface conditions at ramps that fulfil the BLN condition (Bardos et al., 1979).

Since the development of the local ALINEA algorithm by Papageorgiou, many improvements have been proposed among which an adaptive version (Smaragdis et al., 2004). Moreover, some new strategies emerged form the control community such as the switched method proposed in (Sun and Horowitz, 2005) which is based on a controllability analysis. In this paper, we develop a new feedback control approach based on the PieceWise Affine (PWA) formulation of the I-CTM model which enables us to use powerful design methods such as the one proposed in (Ferrari-Trecate et al., 2002). Such feedback controller can be used with the open loop optimal strategy proposed in (Jacquet et al., 2005) to increase it robustness.

\section{MACROSCOPIC TRAFFIC MODELS}

\subsection{The homogeneous LWR model}

The most used macroscopic traffic model is the socalled LWR model (Lighthill and Whitham, 1955) 


$$
\partial_{t} \rho+\partial_{x} \Phi(\rho)=0
$$

where $\rho(t, x)$ is the vehicle density along the freeway section and $\Phi(\cdot)$ is the flux function known as the fundamental diagram. This is a scalar conservation law whose solution $\rho$ may develop discontinuities satisfying the so-called entropy condition (Ansorge, 1990).

An efficient first order numerical method to integrate such equations is the Godunov scheme (Godunov, 1959) that divides the freeway into cells of average density $\rho_{i}$ and applies the stepping

$$
\rho_{i}^{n+1}=\rho_{i}^{n}+\frac{\Delta t}{\Delta x_{i}}\left(\Phi_{n}\left(\rho_{i-1}^{n}, \rho_{i}^{n}\right)-\Phi_{n}\left(\rho_{i}^{n}, \rho_{i+1}^{n}\right)\right)
$$

with $\Phi_{n}\left(\rho_{L}, \rho_{R}\right)$ the flux solution of the Riemann problem (piecewise constant initial condition) with left and right states $\rho_{L}$ and $\rho_{R}$. Though $\Phi_{n}\left(\rho_{L}, \rho_{R}\right)$ may be difficult to compute in general, Daganzo (Daganzo, 1994) showed that the triangular flux function $\Phi(\rho)=\min \left(v \rho, w\left(\rho_{m}-\rho\right)\right)$ with free flow speed $v$, congestion wave speed $w$ and maximal density $\rho_{m}$ leads to the simplified numerical flux formulation

$$
\Phi_{n}\left(\rho_{L}, \rho_{R}\right)=\min \left(v \rho_{L}, w\left(\rho_{m}-\rho_{R}\right), \Phi_{m}\right)
$$

with $\phi_{m}=\max \Phi(\cdot)$ the maximal possible flow. The interest of the CTM model for control design is to be piecewise affine, a property we would like to keep in the inhomogeneous situation.

\subsection{The inhomogeneous LWR model}

One way to model on and off ramps is to consider a concatenation of homogenous links coupled through interface conditions involving their boundary conditions. Nevertheless, such boundary conditions should be handled with care as they should be interpreted in the weak sense (Bardos et al., 1979) as for the LWR equation (Ansorge, 1990). As shown in (Strub and Bayen, 2006), a practical consequence is that boundary conditions do not always apply.

We solve below the interface condition for 2 identical links interconnected through an on-ramp with flow $\hat{\phi}_{i}(t)>0$ as represented on Figure 1. The flow

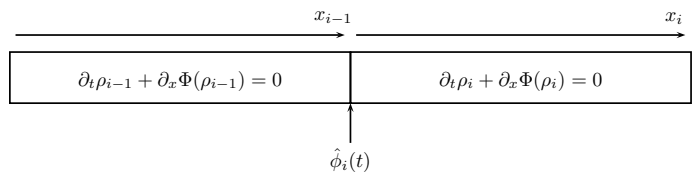

Fig. 1. Interconnected links through an on-ramp.

function $\Phi(\cdot)$ is often taken as a concave function but it can be more general as soon as it satisfies $\Phi(\cdot)>0, \Phi(0)=\Phi\left(\rho_{m}\right)=0, \Phi\left(\rho_{c}\right)=\max \Phi(\cdot)=\Phi_{m}$, $\Phi^{\prime}(\cdot)>0$ on $\left[0, \rho_{c}\right]$ and $\Phi^{\prime}(\cdot)<0$ on $\left[\rho_{c}, \rho_{m}\right]$ with $\rho_{m}$ and $\rho_{c}$ respectively the maximal and critical densities and $\Phi_{m}$ the maximal flow.
The interface flow conservation writes formally

$$
\Phi\left(\rho_{i}^{l}\right)=\Phi\left(\rho_{i-1}^{r}\right)+\hat{\phi}_{i}(t)
$$

with $\rho_{i}^{l}$ and $\rho_{i-1}^{r}$ respectively the left and right boundary values. But (2) cannot be solved in general as $\Phi(\cdot)$ is not invertible (not one-to-one) and has finite range $\left[0, \Phi_{m}\right]$. Moreover, (2) does not embed any causality so it does not tell which boundary value set the other. This problem is solved using the two following arguments:

- The characteristic orientations on both sides of the interface provide the causality.

- (2) is extended by a flow/demand arbitration to ensure its solvability (Lebacque, 1996).

Figures 2 and 3 illustrate the interface behaviors for undercritical and overcritical densities. Such demand/offer arbitration is equivalent to the Finite State Machine (FSM) of Figure 4 with states

(1) free state: $\rho_{i-1}^{r}<\rho_{c}$ and $\rho_{i}^{l}<\rho_{c}$

$$
\rho_{i}^{l}=\Phi^{-l}\left(\Phi\left(\rho_{i-1}^{r}\right)+\hat{\phi}_{i}\right)
$$

with $\Phi^{-l}(\cdot)$ the left inverse of $\Phi(\cdot)$.

(2) congested state: $\rho_{i-1}^{r}>\rho_{c}$ and $\rho_{i}^{l}>\rho_{c}$

$$
\rho_{i-1}^{r}=\Phi^{-r}\left(\Phi\left(\rho_{i}^{l}\right)-\hat{\phi}_{i}\right)
$$

with $\Phi^{-r}(\cdot)$ the right inverse of $\Phi(\cdot)$.

(3) decoupled state: $\Phi\left(\rho_{i-1}^{r}\right)>\Phi_{m}$ or $\rho_{i}^{l}=\rho_{c}$

$$
\left\{\begin{array}{l}
\rho_{i}^{l}=\rho_{c} \\
\rho_{i-1}^{r}=\Phi^{-r}\left(\Phi_{m}-\hat{\phi}_{i}\right)
\end{array}\right.
$$

More states should be considered when solving general Riemann problems but we only consider the most important ones for our application. Note that the congested and decoupled states have jump discontinuity that are not entropic (left value larger than right value). Similarly, a FSM can be built for an off-ramp but it is omitted here.

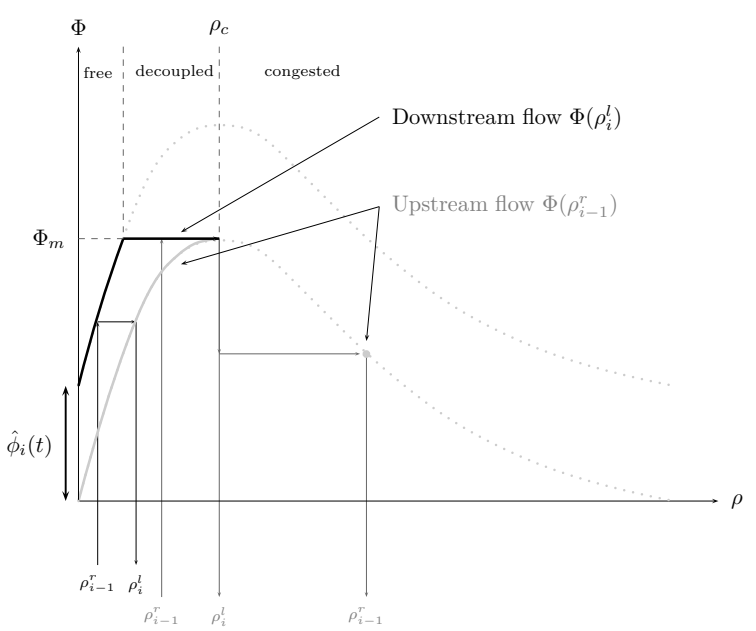

Fig. 2. On-ramp demand/offer arbitration with densities below $\rho_{c}$. The black density computation corresponds to a free interface and the gray computation to a decoupled interface. 


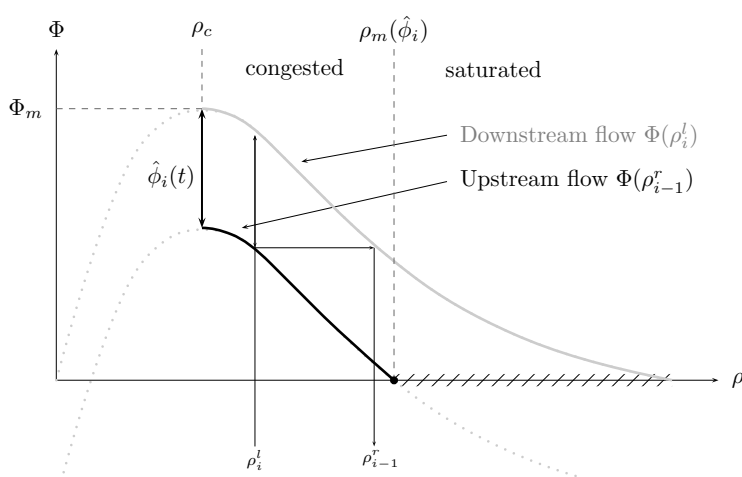

Fig. 3. Arbitration with densities above $\rho_{c}$.

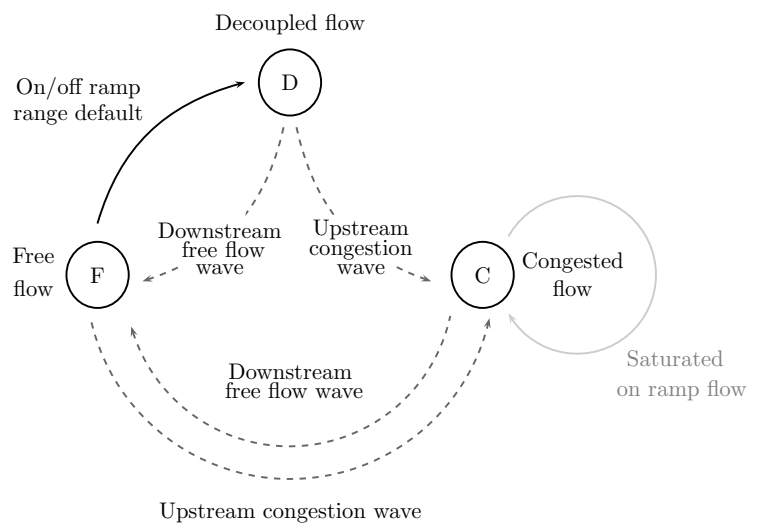

Fig. 4. FSM of an on/off-ramp interface.

As for the homogeneous LWR model, Daganzo's demand-offer-saturation paradigm can be extended to the I-CTM. With $\Phi_{i}^{-}$the flux leaving cell $i-1$ and $\Phi_{i}^{+}$the flux entering cell $i$, the Godunov scheme writes simply

$$
\rho_{i}^{n+1}=\rho_{i}^{n}+c_{i}\left(\Phi_{i}^{+}-\Phi_{i+1}^{-}\right)
$$

where $c_{i}=\Delta t / \Delta x_{i}$ is a discretization parameter. With $r_{i}$ the on-ramp flow and $s_{i}$ the off-ramp flow when present, the numerical fluxes $\Phi^{+}$and $\Phi^{-}$can be written in their most general form

$$
\left\{\begin{aligned}
\Phi_{i}^{+} & =\min \left\{v \rho_{i-1}+r_{i}-s_{i}, w\left(\rho_{m}-\rho_{i}\right), q_{m}-s_{i}\right\} \\
\Phi_{i}^{-} & =\max \left(q_{i}^{+}-r_{i}+s_{i}, 0\right) \\
& \simeq \min \left\{v \rho_{i-1}, w\left(\rho_{m}-\rho_{i}\right)-r_{i}+s_{i}, q_{m}-r_{i}\right\}
\end{aligned}\right.
$$

where the 2 kinds of ramps cannot be present at the same location. Let denote $F_{i}, C_{i}, D_{i}$ the indicator functions that take value 1 when interface $i$ upstream of cell $i$ is respectively free, congested and decoupled. The discretized model becomes

$$
\begin{aligned}
\rho_{i}^{n+1}= & \rho_{i}^{n}+c_{i} v \rho_{i-1}^{n} F_{i}+c_{i} r_{i} F_{i}-c_{i} s_{i} F_{i} \\
& +c_{i} q_{m} D_{i}-c_{i} s_{i} D_{i}+c_{i} w\left(\rho_{m}-\rho_{i}^{n}\right) C_{i} \\
& -c_{i} v \rho_{i}^{n} F_{i+1}-c_{i} q_{m} D_{i+1}+c_{i} r_{i+1} D_{i+1} \\
& -c_{i} w\left(\rho_{m}-\rho_{i+1}^{n}\right) C_{i+1}+c_{i} r_{i+1} C_{i+1}
\end{aligned}
$$

with slight changes at boundaries. Reordering in

$$
\begin{aligned}
& \rho_{i}^{n+1}=\underbrace{\left(c_{i} F_{i}\right) r_{i}+\left(c_{i} C_{i+1}+c_{i} D_{i+1}\right) r_{i+1}}_{B \text { matrix }}+\underbrace{\left(-c_{i} F_{i}-c_{i} D_{i}\right) s_{i}}_{W \text { matrix }} \\
& +\underbrace{\left(c_{i} v F_{i}\right) \rho_{i-1}^{n}+\left(1-c_{i} w C_{i}-c_{i} v F_{i+1}\right) \rho_{i}^{n}+\left(c_{i} w C_{i+1}\right) \rho_{i+1}^{n}}_{A \text { matrix }} \\
& +\underbrace{\left(c_{i} D_{i}-c_{i} D_{i+1}\right) q_{m}+\left(c_{i} w C_{i}-c_{i} w C_{i+1}\right) \rho_{m}}_{a \text { vector }}
\end{aligned}
$$

the interest of this formulation is that it can be put in a switched piecewise affine (PWA) form suitable for controller design

$$
\left\{\begin{array}{l}
\rho_{k+1}=A_{\alpha_{k}} \rho_{k}+B_{\alpha_{k}} u_{k}+W_{\alpha_{k}} w_{k}+a_{\alpha_{k}} \\
\alpha_{k}=g\left(\rho_{k}, u_{k}, w_{k}\right)
\end{array}\right.
$$

$\alpha_{k}=\left(d_{k}^{1}, \ldots, d_{k}^{n+1}\right) \in \mathcal{I}$ is the discrete state where $d_{k}^{i}=\{\mathrm{F}, \mathrm{C}, \mathrm{D}\}$ is the $i^{t h}$ interface state, $d_{k}^{1}$ the upstream boundary state and $d_{k}^{n+1}$ the downstream boundary state. $\rho=\left(\rho_{1}, \ldots, \rho_{n}\right)^{T}$ denotes the vector of cell densities, $r=\left(r_{1}, \ldots, r_{m}\right)^{T}$ the vector of on-ramp flows, $w_{k}=\left(s_{k}, \rho_{k}^{\mathrm{Up}}, \rho_{k}^{\mathrm{Do}}\right)^{T}$ the vector of partially unknown exogenous signals and $a_{\alpha_{k}}$ is an affine term. $g\left(\rho_{k}, u_{k}, w_{k}\right)$ is a nonlinear function that returns the discrete state corresponding to $\Phi_{i}^{+}$. The discrete state $\alpha_{k}$ that depends on this switching rule sets the active matrices valid for the state space representation at time $k$

$$
\begin{array}{ll}
A_{\alpha_{k}} \in\left\{A_{1}, \ldots, A_{h}\right\} & B_{\alpha_{k}} \in\left\{B_{1}, \ldots, B_{h}\right\} \\
W_{\alpha_{k}} \in\left\{W_{1}, \ldots, W_{h}\right\} & a_{\alpha_{k}} \in\left\{a_{1}, \ldots, a_{h}\right\}
\end{array}
$$

In this discrete model, which has some similarity with (Sun and Horowitz, 2005), the switches play the role of the nonlinearity of the continuous one.

\section{METERING CONTROLLER DESIGNS}

With the objective to track the reference $\left(\bar{u}_{k}, \bar{\rho}_{k}\right)$ and assuming identical sequences $\alpha_{k}=\bar{\alpha}_{k}$, the error dynamics for the continuous state is

$$
\tilde{\rho}_{k+1}=A_{\alpha_{k}} \tilde{\rho}_{k}+B_{\alpha_{k}} \tilde{u}_{k}+W_{\alpha_{k}} \tilde{w}_{k}
$$

Let consider the problem of designing a piecewise linear full state controller $\tilde{u}_{k}=K_{\alpha_{k}} \tilde{\rho}_{k}$ giving the following closed loop continuous dynamics

$$
\tilde{\rho}_{k+1}=\left(A_{\alpha_{k}}+B_{\alpha_{k}} K_{\alpha_{k}}\right) \tilde{\rho}_{k}+W_{\alpha_{k}} \tilde{w}_{k}
$$

The closed loop system to stabilize thus writes

$$
\left\{\begin{array}{l}
\tilde{\rho}_{k+1}=\Pi_{\alpha_{k}} \tilde{\rho}_{k}+W_{\alpha_{k}} \tilde{w}_{k} \\
\alpha_{k}=g\left(\bar{\rho}_{k}+\tilde{\rho}_{k}, \bar{u}_{k}+\tilde{u}_{k}, \bar{w}_{k}+\tilde{w}_{k}\right) \\
z_{k}=C_{\alpha_{k}} \rho_{k}
\end{array}\right.
$$

with $C_{\alpha_{k}}$ weighting the states to be controlled.

In (Ferrari-Trecate et al., 2002), the authors proposed to use passivity theory to design feedback controller for PWA systems. System (8) is strictly passive with supply rate $W: \mathbb{R}^{q} \times \mathbb{R}^{p} \rightarrow \mathbb{R}$ if there exists a non negative storage function of the form $V: \mathcal{I} \times \mathbb{R}^{n} \rightarrow \mathbb{R}$ with $V(\cdot, 0)=0$ such that the following dissipation inequality holds

$$
\forall w, \forall k, \quad \Delta V_{k}=V_{k+1}-V_{k}<W\left(z_{k}, w_{k}\right)
$$

where $V_{k}=V\left(\alpha_{k}, \tilde{\rho}_{k}\right)$. The following supply rates are classical in controller design

$$
\begin{aligned}
& W_{\text {stab }}=0 \\
& W_{\infty}=\gamma^{2} w_{k}^{T} w_{k}-z_{k}^{T} z_{k} \\
& W_{L Q}=W_{2}=-z_{k}^{T} Q z_{k}
\end{aligned}
$$

$W_{\text {stab }}$ defines Lyapunov stabilization criteria, $W_{\infty}$ defines the $\mathcal{H}_{\infty}$ perturbation attenuation criteria 
and $W_{L Q}$ the $L Q$ performance criteria, whose special case $Q=I$ corresponds to the $\mathcal{H}_{2}$ norm.

For PWA systems, a candidate storage function is the piecewise quadratic (PWQ) Lyapunov function defined by

$$
V_{k}=V\left(\alpha_{k}, \tilde{\rho}_{k}\right)=\tilde{\rho}_{k}^{T} P_{\alpha_{k}} \tilde{\rho}_{k} \text { with } P_{i}^{T}=P_{i}>0
$$

where the matrices $P_{i}$ are considered symmetric without loss of generality. The decrease in the storage function along the system trajectory is

$$
\begin{aligned}
& \Delta V_{k}= \\
& V\left(\alpha_{k+1}, \tilde{\rho}_{k+1}\right)-V\left(\alpha_{k}, \tilde{\rho}_{k}\right)= \\
& \tilde{\rho}_{k+1}^{T} P_{\alpha_{k+1}} \tilde{\rho}_{k+1}-\tilde{\rho}_{k}^{T} P_{\alpha_{k}} \tilde{\rho}_{k}= \\
& \left(\tilde{\rho}_{k}^{T} \Pi_{\alpha_{k}}^{T}+\tilde{w}_{k}^{T} W_{\alpha_{k}}^{T}\right) P_{\alpha_{k+1}}\left(\Pi_{\alpha_{k}} \tilde{\rho}_{k}+W_{\alpha_{k}} \tilde{w}_{k}\right)-\tilde{\rho}_{k}^{T} P_{\alpha_{k}} \tilde{\rho}_{k}= \\
& \left(\begin{array}{c}
\tilde{\rho}_{k} \\
\tilde{w}_{k}
\end{array}\right)^{T}\left(\begin{array}{cc}
\Pi_{\alpha_{k}}^{T} P_{\alpha_{k+1}} \Pi_{\alpha_{k}}-P_{\alpha_{k}} & \Pi_{\alpha_{k}}^{T} P_{\alpha_{k+1}} W_{\alpha_{k}} \\
W_{\alpha_{k}}^{T} P_{\alpha_{k+1}} \Pi_{\alpha_{k}} & W_{\alpha_{k}}^{T} P_{\alpha_{k+1}} W_{\alpha_{k}}
\end{array}\right)\left(\begin{array}{c}
\tilde{\rho}_{k} \\
\tilde{w}_{k}
\end{array}\right)
\end{aligned}
$$

which simplifies without uncertainties $\left(w_{k}=0\right)$ to

$$
\Delta V_{k}=\tilde{\rho}_{k}^{T}\left(\Pi_{\alpha_{k}}^{T} P_{\alpha_{k+1}} \Pi_{\alpha_{k}}-P_{\alpha_{k}}\right) \tilde{\rho}_{k}
$$

\subsection{Feedback Stabilization Without Uncertainties}

A sufficient condition of global stability for the PWA system (8) without uncertainties $\left(w_{k}=0\right)$ is that $\Delta V\left(\alpha_{k}, \tilde{\rho}_{k}\right)$ is negative definite along the system trajectories. Considering all the possible discrete state trajectories in Equation (10), i.e. either $\alpha_{k+1}=\alpha_{k}$ or $\alpha_{k+1} \neq \alpha_{k}$ for transitions, global stability is obtained if one can find a set of symmetric positive definite matrices $P_{i}$ and constant vector gains $K_{i}$ such that

$$
P_{i}-\Pi_{i}^{T} P_{j} \Pi_{i}>0 \quad, \quad \forall i \rightarrow j
$$

Theorem 1. If there exists symmetric positive definite matrices $Q_{i}=Q_{i}^{T}>0$ and matrices $U_{i}$ of appropriate dimension satisfying the set of Linear Matrix Inequalities (LMI)

$$
\left(\begin{array}{cc}
Q_{i} & \star \\
A_{i} Q_{i}+B_{i} U_{i} & Q_{j}
\end{array}\right)>0 \quad \forall(i, j) \in \mathcal{T}
$$

for all possible transitions $\mathcal{T}$ of the discrete state then $\tilde{\rho}$ converges globally towards the origin with the piecewise linear static gain $K_{i}=U_{i} Q_{i}^{-1}$.

PROOF. We multiply by $P_{i}^{-1}$ from the left and right in (11) to get by congruence

$$
P_{i}^{-1}-P_{i}^{-1} \Pi_{i}^{T} P_{j} \Pi_{i} P_{i}^{-1}>0
$$

which develops as

$$
P_{i}^{-1}-P_{i}^{-1}\left(A_{i}^{T}+K_{i}^{T} B_{i}^{T}\right) P_{j}\left(A_{i}+B_{i} K_{i}\right) P_{i}^{-1}>0
$$

Making the change of variables $Q_{i}=P_{i}^{-1}$ and $U_{i}=K_{i} P_{i}^{-1}$, we get with $\left(P_{i}^{-1}\right)^{T}=P_{i}^{-1}$

$$
Q_{i}-\left(Q_{i} A_{i}^{T}+U_{i}^{T} B_{i}^{T}\right) Q_{j}^{-1}\left(A_{i} Q_{i}+B_{i} U_{i}\right)>0
$$

The schur complement gives the theorem.
The feasibility problem for the LMI (12) requires $\left(A_{i}, B_{i}\right)$ to be stabilizable. Note that the size of the LMI constraint (12) depends directly on the number of transitions $(i, j)$ considered in the set $\mathcal{T}$. Plant augmentation methods can be used to design a PI controller.

\section{2 $\mathcal{H}_{\infty}$ synthesis for perturbation attenuation}

In this section, some robustness requirements are added to the control problem. We consider here an $\mathcal{H}_{\infty}$ problem which consists in minimizing or bounding to a predefined value $\gamma$ the system gain between $\left\|\tilde{w}_{k}\right\|_{2}$ and $\left\|\tilde{\rho}_{k}\right\|_{2}$ so that the influence of the exogenous signal $w$ on the state $\rho$ is controlled.

The supply rate $W_{\infty}$ can be rewritten

$$
\begin{aligned}
W_{\infty}\left(\tilde{\rho}_{k}, \tilde{w}_{k}\right) & =\gamma^{2} \tilde{w}_{k}^{T} \tilde{w}_{k}-\tilde{\rho}_{k}^{T} \tilde{\rho}_{k} \\
& =\left(\begin{array}{c}
\tilde{\rho}_{k} \\
\tilde{w}_{k}
\end{array}\right)^{T}\left(\begin{array}{cc}
-I & 0 \\
0 & \gamma^{2} I
\end{array}\right)\left(\begin{array}{c}
\tilde{\rho}_{k} \\
\tilde{w}_{k}
\end{array}\right)
\end{aligned}
$$

Applying the S-procedure to the passivity inequality (9) and considering the uncertainties in (10), we get the classical Bounded Real Lemma which states that $\left\|\tilde{\rho}_{k}\right\|_{2}<\gamma\left\|\tilde{w}_{k}\right\|_{2}$ is equivalent to

$$
\left(\begin{array}{cc}
\Pi_{\alpha_{k}}^{T} P_{\alpha_{k+1}} \Pi_{\alpha_{k}}-P_{\alpha_{k}}+I & \Pi_{\alpha_{k}}^{T} P_{\alpha_{k+1}} W_{\alpha_{k}} \\
W_{\alpha_{k}}^{T} P_{\alpha_{k+1}} \Pi_{\alpha_{k}} & W_{\alpha_{k}}^{T} P_{\alpha_{k+1}} W_{\alpha_{k}}-\gamma^{2} I
\end{array}\right)<0
$$

Theorem 2. The attenuation $\left\|\tilde{\rho}_{k}\right\|_{2}<\gamma\left\|\tilde{w}_{k}\right\|_{2}$ is realized by the static feedback gains $K_{i}$ for all signals $\tilde{w}_{k}$ in $l_{2}$ if one can find a set of matrices $Q_{i}=Q_{i}^{T}>0$ and $R_{i}$ such that

$$
\left(\begin{array}{cccc}
Q_{i} & \star & \star & \star \\
0 & \gamma^{2} I & \star & \star \\
A_{i} Q_{i}+B_{i} R_{i} & W_{i} & Q_{j} & \star \\
Q_{i} & 0 & 0 & I
\end{array}\right)>0 \quad \forall(i, j) \in \mathcal{T}
$$

with $\mathcal{T}$ the set of possible transitions. The feedback gains are given by $K_{i}=U_{i} Q_{i}^{-1}$.

PROOF. By congruence of (13) with $\operatorname{diag}\left(P_{k}^{-1}, I\right)$ and by setting $Q_{k}=P_{k}^{-1}$, we have

$$
\left(\begin{array}{cc}
Q_{k} \Pi_{k}^{T} P_{k+1} \Pi_{k} Q_{k}-Q_{k}+Q_{k}^{T} Q_{k} & Q_{k} \Pi_{k}^{T} P_{k+1} W_{k} \\
W_{k}^{T} P_{k+1} \Pi_{k} Q_{k} & W_{k}^{T} P_{k+1} W_{k}-\gamma^{2} I
\end{array}\right)<0
$$

which can be rewritten

$\left(\begin{array}{cc}Q_{k} & 0 \\ 0 & \gamma^{2} I\end{array}\right)-\left(\begin{array}{cc}\Pi_{k} Q_{k} & W_{k} \\ Q_{k} & 0\end{array}\right)^{T}\left(\begin{array}{cc}P_{k+1} & 0 \\ 0 & I\end{array}\right)^{T}\left(\begin{array}{cc}\Pi_{k} Q_{k} & W_{k} \\ Q_{k} & 0\end{array}\right)>0$

The Schur complement Lemma then gives

$$
\left(\begin{array}{cccc}
Q_{k} & 0 & Q_{k} \Pi_{k}^{T} & Q_{k} \\
0 & \gamma^{2} I & W_{k}^{T} & 0 \\
\Pi_{k} Q_{k} & W_{k} & Q_{k+1} & 0 \\
Q_{k} & 0 & 0 & I
\end{array}\right)>0
$$

Setting $U_{k}=K_{k} Q_{k}$, the nonlinear term becomes $\Pi_{k} Q_{k}=A_{k} Q_{k}+B_{k} U_{k}$, giving the theorem. 


\section{SIMULATION RESULTS}

\subsection{Study case of Lyon South-Est beltway}

Let consider as a study case the section of the South-Est beltway of Lyon (France) depicted in Figure 5. As shown in this figure, velocity time series (provided by Direction Départemental de l'Équipement 69) at different mainlane locations can be used to determine which on-ramp is responsible of the congestion propagating upstream. Having identified the bottleneck on Lyon's South-

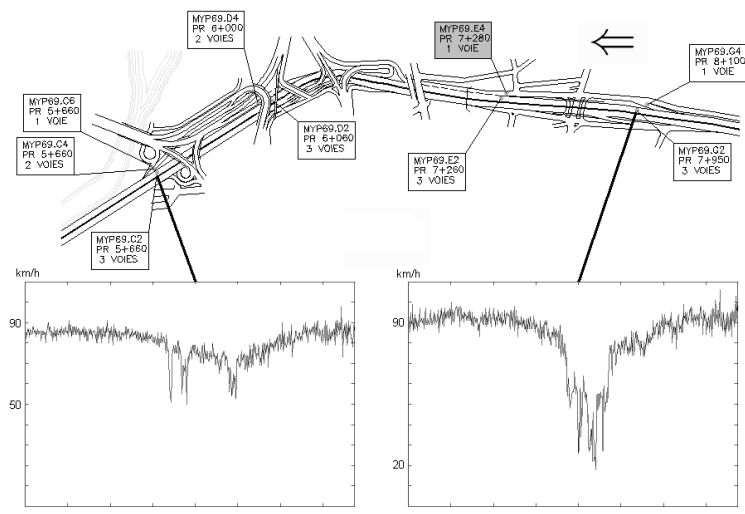

Fig. 5. Freeway section treated in the example where the arrow indicates the considered traffic direction and the gray dots the locations of the labelled inductive loops. The plotted velocity time series on 18/10/2005 from 11:00 to $23: 59$ show that the on-ramp with shaded label is responsible of the congestion.

Est beltway, the surrounding freeway is modelled as a concatenation of 3 homogeneous links interconnected through an on-ramp and an off-ramp. Figure 6 shows how this section looks in the Matlab $^{\circledR}$ Simulink ${ }^{\circledR}$ toolbox we developed for inhomogeneous macroscopic freeway modelling. The first

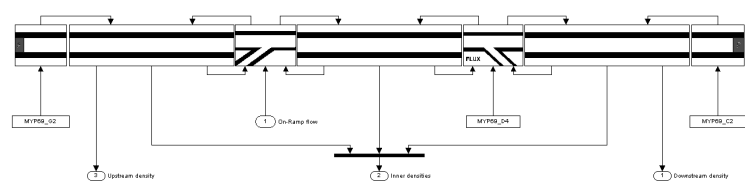

Fig. 6. Abstract model of Lyon freeway section.

link is discretized into 2 cells and the 2 others are considered to be 1 cell. The boundary data and the off-ramp flow are provided by the density time series measured at the corresponding loops. All the cell density are assumed available in the feedback controller design.

As mentioned earlier, the possible discrete state transitions should be identified before the controller design. The on-ramp responsible of the congestion being on the third interface, the possible transitions are given by

$$
F F F F F \Leftrightarrow F F D F F \Leftrightarrow F C D F F \Leftrightarrow C C D F F
$$

For instance, the matrices of FFDFF are

$$
\begin{gathered}
A(C C D F F)=\left(\begin{array}{cccc}
1-w_{1} c_{1} & w_{2} c_{1} & 0 & 0 \\
0 & 1-w_{2} c_{2} & 0 & 0 \\
0 & 0 & 1-v_{3} c_{3} & 0 \\
0 & 0 & v_{3} c_{4} & 1-v_{4} c_{4}
\end{array}\right) \\
B(C C D F F)=\left(\begin{array}{c}
0 \\
c_{2} \\
0 \\
0
\end{array}\right) \quad W(C C D F F)=\left(\begin{array}{ccc}
0 & 0 & 0 \\
0 & 0 & 0 \\
0 & 0 & 0 \\
-c_{4} & 0 & 0
\end{array}\right) \\
a(C C D F F)=\left(\begin{array}{c}
c_{1} w_{1} \rho_{m 1}-c_{1} w_{2} \rho_{m 2} \\
c_{2} w_{2} \rho_{m 2}-c_{2} \Phi_{m 3} \\
c_{3} \Phi_{m 3} \\
0
\end{array}\right)
\end{gathered}
$$

Similar matrices are computed for all of the 4 discrete state considered in the example.

\subsection{Numerical experiments}

The LMIs obtained in section 3 for the stabilizing controller have been coded in the Matlab ${ }^{\circledR}$ LMI Control Toolbox. These LMIs being feasible for the Lyon beltway study case, we were able to compute the feedback gains that stabilize the freeway error dynamics.

The choice of a suitable density reference is an important task that should not be underestimated. Though the critical density corresponds to the maximal flow, it should not be taken as the reference as it may lead to unrealistically large onramp queues. Instead, the freeway should be allowed to be partially congested due to the unavoidable excess demand. This objective is met by requiring a minimum on-ramp flow that keeps ramp queues at a reasonable level. The simulations shown below have a minimal on-ramp flow of $1100 \mathrm{veh} / \mathrm{h}$.

Figure 7 shows the efficiency of the feedback method with a simulation from $15 \mathrm{~h} 30$ to $22 \mathrm{~h} 00$ and a congestion from $17 \mathrm{~h} 30$ to $20 \mathrm{~h} 00$. Figure 8 shows the demand and the resulting on-ramp queue. As could be expected, reducing the minimum on-ramp flow in our reference increases the peak ramp queue. The computed feedback gains are provided in Figure 9 for all discrete states.

An interesting observation is that the obtained feedback gains are local, implying that local algorithm such as Alinea are sufficient when a single on-ramp is metered. Moreover, as the dominant coefficient moves from the cell downstream of the ramp to the one upstream of it depending on the discrete state, a switched version of Alinea as the one proposed in (Sun and Horowitz, 2005) should be considered in local ramp metering strategies. 


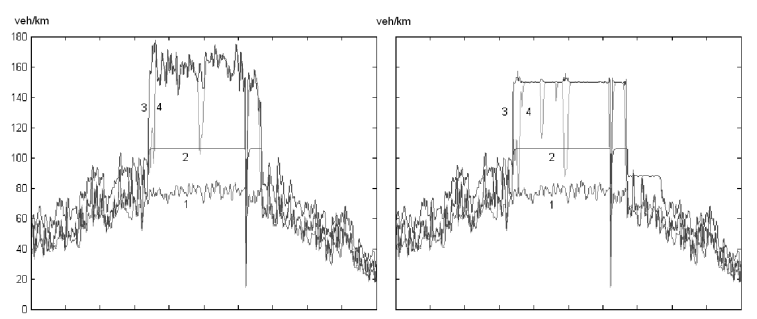

Fig. 7. Comparison of the density time series at cells 1, 2, 3 and 4 without (left) and with (right) ramp metering when a minimum of $1100 \mathrm{veh} / \mathrm{h}$ is required at the on-ramp.

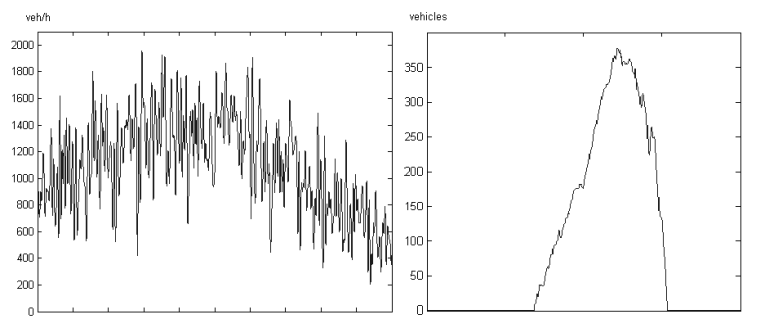

Fig. 8. Demand at the on-ramp (left) and queue length (right) with $1100 \mathrm{veh} / \mathrm{h}$ allowed.
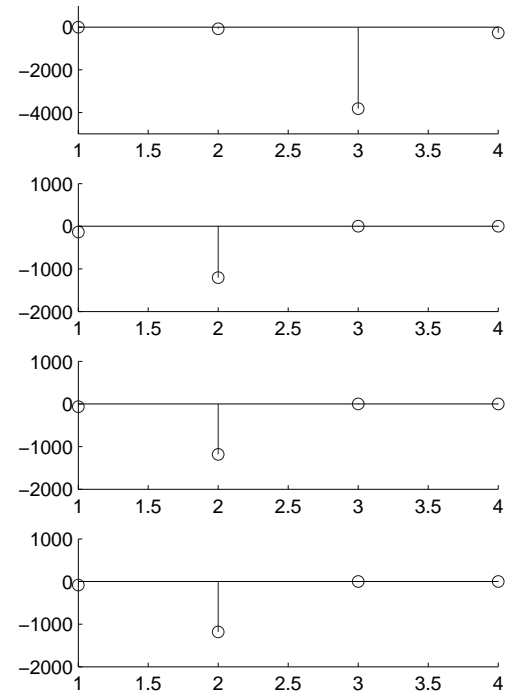

Fig. 9. Feedback gains of the 4 cells for the discrete state FFFFF (top), FFDFF, FCDFF and $C C D F F$ (bottom).

\section{CONCLUSION}

We proposed in this paper an extension of Daganzo's CTM discretization to inhomogeneous freeways that can be put in the form of a piecewise affine system. Based on this model, an LMI-based controller synthesis were proposed, both for stabilization and $\mathcal{H}_{\infty}$ control. A simulation with real traffic data shows the efficiently of the proposed method that may be used for coordinated ramp metering. A software that integrates these algorithms is currently under development.

\section{REFERENCES}

Ansorge, R. (1990). What does the entropy condition mean in traffic flow theory?. Transportation Research Part B: Methodological.

Bardos, C., A.Y. LeRoux and J.C. Nedelec (1979). First order qusilinear equations with bounday conditions. Communications in Partial Differential Equations.

Daganzo, C. F. (1994). The cell transmission model: A dynamic representation of highway traffic consistent with the hydrodynamic theory. Transportation Research Part B: Methodological.

Ferrari-Trecate, G., F.A. Cuzzola, D. Mignone and M. Morari (2002). Analysis of discretetime piecewise affine and hybrid systems. Automatica.

Godunov, S. (1959). A difference method for the numerical calculation of discontinuous solutions of hydrodynamic equations. Matematicheskii Sbornik.

Jacquet, D., C. Canudas de Wit and D. Koenig (2005). Traffic control and monitoring with a macroscopic model in the presence of strong congestion waves. In: Proceedings of the 2005 Conference on Decision and Control.

Lebacque, J.P. (1996). The godunov scheme and what it means for first order traffic flow models. In: Proceedings of the ISTTT Conference.

Levinson, D. and L. Zhang (2005). Meters on trial: Evidence from the twin cities metering holiday. Transportation Research Part A: Policy and Practice.

Lighthill, M.J. and G.B. Whitham (1955). On kinematic waves II: a theory of traffic flow on long crowded roads. In: Proceedings Royal Society, London Series A.

Papageorgiou, M., H. Haj-Salem and F. Middelham (1997). Alinea local ramp metering: Summary of field results. Transportation Research Record.

Smaragdis, E., M. Papageorgiou and E. Kosmatopoulos (2004). A flow-maximizing adaptive local ramp metering strategy. Transportation Research Part B: Methodological.

Strub, I. and A. Bayen (2006). Weak formulation of hybrid boundary conditions for scalar conservation laws: an application to highway modeling. In: Proceedings Hybrid Systems Computation and Control.

Sun, X. and R. Horowitz (2005). Localized switching ramp-metering controller with queue length regulator for congested freeways. In: Proceedings of the IEEE 2005 American Control Conference. 